\title{
Typical versus atypical antipsychotics: Genotoxicity and cytostaticity testing
}

\author{
Evangelos G Katsioulis, Maria Argyraki, Maria T Ekonomopoulou*, Constantine Mourelatos, Zafiroula Iakovidou-Kritsi and Dionysios \\ Mourelatos
}

Laboratory of Medical Biology and Genetics, School of Medicine, Aristotle University of Thessaloniki, Greece

\begin{abstract}
Cultured human lymphocytes were treated with two typical (Levomepromazine/LVP and Perphenazine/PRZ) and two atypical (Amisulpride/AMD and Sulpiride/ SPD) antipsychotics at concentrations equivalent to the oral doses used in clinical praxis. Sister Chromatid Exchanges (SCE) were evaluated as indicators of genotoxicity and Proliferation Rate Index (PRI) and Mitotic Index (MI) were measured as indicators of cytostatic effect. Typical and atypical antipsychotics were identified as significantly $(\mathrm{p}<0.01)$ elevating SCE frequencies and significantly depressing PRI $(\mathrm{p}<0.01)$ and $\mathrm{MI}(\mathrm{p}<0.01)$. A significant correlation ( $<<0.01)$ was also observed between the magnitude of the SCE response and the PRI depression achieved by the typical and the atypical antipsychotics tested. The hypothesis of a possible antineoplastic potential of these compounds is discussed.
\end{abstract}

Abbreviations: LVZ: Levomepromazine, PRZ: Perphenazine, AMS: Amisulpride, SPD: Sulpiride, SCEs: Sister Chromatid Exchanges, PRI: Proliferation Rate Index, MI: Mitotic Index

\section{Introduction}

Antipsychotics or major tranquilizers are a class of drugs used primarily to manage schizophrenia and bipolar disorder. They act mainly as dopamine D2 antagonists and thus improve disorders of thinking, perception, emotion, hyperkinetic behavior. Antipsychotics can be classified in typical and atypical [1]. Due to their wide range of chemical structure and extensive use they are being investigated worldwide for side/adverse effects and/or involvement in other diseases.

Levomepromazine ((2R)-3-(2-methoxyphenothiazin-10-yl)-N,N,2 trimethylpropan-1-amine/Figure A), also known as methotrimeprazine, is an aliphatic phenothiazine with low antipsychotic potency first used in psychiatry for the treatment of schizophrenia [2]. Levomepromazine (LVZ) acts as an antagonist at histamine-1, muscarinic-cholinergic, dopaminergic-2, alpha-1 adrenergic and 5HT-2 receptors [3]. In palliative care, $\mathrm{LVZ}$ is predominantly used for the treatment of nausea and vomiting, for severe delirium or agitation at the end of life and as an analgesic for moderate to severe pain in non-ambulant patients [4]. Known adverse effects of LVZ include postural hypotension, skin irritation, drowsiness, dry mouth, dystonia, neuroleptic malignant syndrome, Parkinsonism and epilepsy by lowering the seizure threshold [5].

Perphenazine (2-[4-[3-(2-chlorophenothiazin-10-yl)propyl]piperazin-1-yl]ethanol/Figure B) is a piperazinyl phenothiazine and a dopamine antagonist with antiemetic and antipsychotic properties [6]. Perphenazine (PRZ) blocks postsynaptic dopamine 2(D2) receptors in the mesolimbic and medullary chemoreceptor trigger zone (CTZ), thereby preventing the excess of dopamine in the brain. This leads to reduction in psychotic symptoms, such as hallucinations and delusions. PRZ appears to exert its antiemetic activity by blocking the dopamine and histamine- 1 receptor in the CTZ, thereby relieving nausea and vomiting in the brain [7]. Its main indication is the treatment of schizophrenia, but it is also used for other psychiatric disorders such as mania, agitated behaviour and severe anxiety as well as for the management of post-operative or chemotherapy-induced nausea and vomiting and intractable hiccup. PRZ causes early and late extrapyramidal side effects. When used for its strong antiemetic or antivertigo effects in cases with associated brain injuries, it may obscure the clinical course and interferes with the diagnosis. High doses of PRZ can cause temporary dyskinesia. As with other typical antipsychotics, permanent or lasting tardive dyskinesia is a risk [8]

Amisulpride (4-amino- N-[(1-ethylpyrrolidin- 2-yl) methyl] -5-ethylsulfonyl-2-methoxybenzamide/Figure C) is a substituted benzamide derivative with analgesic, antiemetic and antipsychotic effects [9]. Amisulpride (AMD) exerts its effects acting on various neurotransmitter receptors in the CNS. Its antipsychotic effects include antagonism of the $\mathrm{D}_{2} / \mathrm{D}_{3}$ dopamine receptor. Its potent antagonism of the $5-\mathrm{HT}_{7}$ serotonin receptor causes its antidepressant effects. AMD binds and activates the gamma-hydroxybutyrate GHB receptor [10]. There is a strong binding affinity of AMD with the $5-\mathrm{HT}_{2 \mathrm{~B}}$ serotonin receptor, which is yet of undetermined clinical interpretation [11]. It is approved for the treatment of psychosis and schizophrenia in Europe, Asia and Australia, however it is not approved by FDA for use in USA. Based on available reports, AMD has a high prescription rate among the antipsychotic agents [12]. Besides the confirmed antipsychotic properties, AMD is reported to have several adverse effects, such as neuroleptic malignant syndrome, extrapyramidal side-effects, nausea, agitation, weight gain and metabolic disturbances [13].

*Correspondence to: Maria T Ekonomopoulou, Laboratory of Medical Biology and Genetics, School of Medicine, Aristotle University of Thessaloniki, 54124, Hellas, Greece, Tel/Fax: 00302310430131, E-mail: oikonomomar@gmail.com

Key words: antipsychotics, genotoxicity, cytotoxicity, SCEs, PRI, antineoplastic

Received: June 05, 2020; Accepted: June 23, 2020; Published: June 26, 2020 
<smiles>COc1ccc2c(c1)N(C[C@@H](C)CN(C)C)c1ccccc1S2</smiles>

Figure A: Levopromazine<smiles>OCCN1CCN(CCCN2c3ccccc3Sc3ccc(Cl)cc32)CC1</smiles>

Figure B: Perphenazine

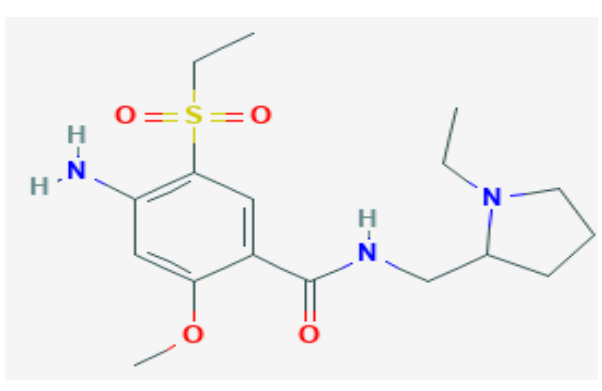

Figure C: Amisulpride

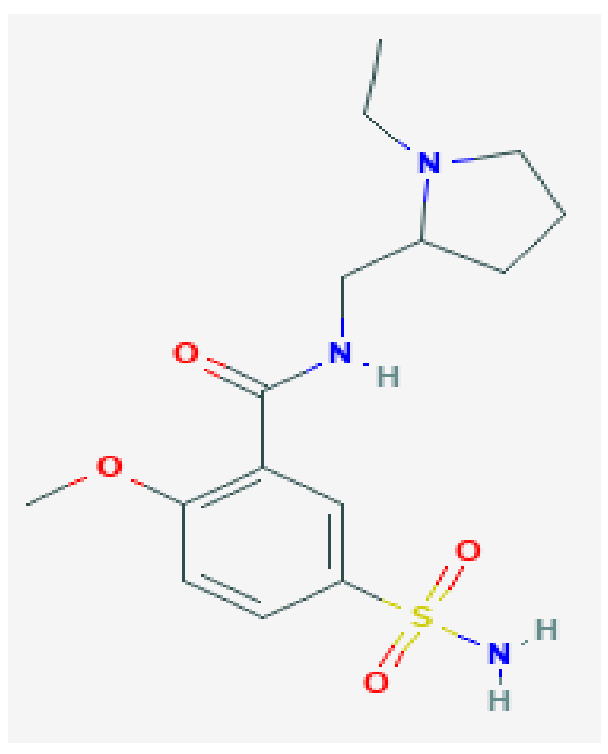

Figure D: Sulpiride
Sulpiride (N-[(1-ethylpyrrolidin-2-yl)methyl]-2-methoxy-5- sulfamoylbenzamide/Figure D) is a benzamide atypical antipsychotic drug used mainly in the treatment of psychosis associated with schizophrenia and major depressive disorder. In some cases Sulpiride (SPD) is used in low dosage to treat anxiety and mild depression [14]. It is chemically and clinically similar to Amisulpride. SPD is a selective antagonist of D2 and D3 dopamine receptors. This effect dominates in doses exceeding $600 \mathrm{mg}$ daily. In doses of 600 to $1.600 \mathrm{mg}$ SPD exhibits mild sedating and antipsychotic activity. In low doses (in particular 50 to $200 \mathrm{mg}$ daily) its prominent feature is antagonism of presynaptic inhibitory dopamine receptors accounting for some antidepressant activity and a stimulating effect. Therefore, it is in these doses used as a second line antidepressant. Additionally, it alleviates vertigo [15]. The benzamide neuroleptics (including Sulpiride, Amisulpride and Sultopride) have been shown to activate the endogenous gamma-hydroxybutyrate receptor in vivo at therapeutic concentrations [16]. The most common side effects of SPD are dizziness, headache, extrapyramidal side effects insomnia, nausea, vomiting, nasal congestion, anticholinergic adverse effects [17].

There is evidence that antipsychotics exhibit genotoxic and carcinogenic or cytostatic effects $[18,19]$. In a previous study of our group, we examined the genotoxic and cytotoxic effect of Valproic Acid and Ziprasidone [20]. In the current study we decided to use Levomepromazine (LVZ) and Perphenazine (PRZ) as typical antipsychotics and Amisulpride (AMD) and Sulpiride (SPD) as atypical ones. The genotoxic effect was investigated by the induction of sister chromatid exchanges (SCEs) in normal human lymphocyte cultures of peripheral blood and for the cytostatic activity we estimated the Proliferation Rate Index (PRI) and the Mitotic Index (MI).

Many phenothiazines have been studied for their cytotoxic activity. Most of them inhibit cell proliferation, which could be used in cancer therapy [21-26]. Chloropromazine is one the most studied antipsychotics and it has been found cytotoxic and antineoplastic in healthy and cancer cells respectively [27-29]. There is very limited data on the cytogenetic activity of LVZ. As far as PRZ is concerned, cytotoxic and cytostatic activity has been reported in human lymphocytes and cancer cells [30].

Although first generation antipsychotics have been studied for their cytogenetic activities, similar studies are very limited regarding second generation antipsychotics [19]. There is evidence indicating that Amisulpride (AMD) has potential cytotoxic effects on human white blood cells being involved in the development of leukopenia and neutropenia [31]. Sulpiride (SPD) has also been investigated for its cytotoxicity. In a study, SPD caused a dose-dependent reduction of TNF-a's (Tumor Necrosis Factor-a) levels of peritoneal mice' macrophages [31]. SPD, along with Clozapine, has been found to activate DNA demethylation in the brain $[32,33]$.

The consequences of the exposure to potential mutagenscarcinogens have been commonly studied in humans by cytogenetic methods, and positive responses should be regarded as indicators of a genotoxic hazard. The CA assay is used as a sensitive biomarker of genotoxicity, but SCE frequency has been proved as one of the most sensitive indices for detecting genotoxicity. PRI has been established as a valuable indicator of cytostatic effect. Lately, the estimation of the relationship between SCE induction and PRI depression has been proposed as one of the methods for evaluating chemotherapeutic efficiency in vitro and in vivo [34]. SCEs can provide insight in the 
cytogenetic damage induced by various genotoxic agents at very low concentrations. Moreover, the determination of proliferation rates in lymphocyte cultures has been proved to be a very useful and sensitive indicator of the cytostatic action of various environmental hazards or therapeutic agents [35].

\section{Materials and methods}

The cytogenetic effects, at six concentrations for each drug, were investigated in the lymphocytes of 3 healthy young donors (for each drug), who were not taking medication, smoking or consuming alcohol. All donors became acquainted with the study, and willingly signed permission slips to allow the use of their blood samples for scientific purposes. The concentrations of the drugs were equivalent to the oral doses used in clinical praxis. The $2.5 \mu \mathrm{g} / \mathrm{ml}$ dose of $\mathrm{LPZ}$ corresponds to approximately $12.5 \mathrm{mg} \mathrm{LPZ} \mathrm{for} \mathrm{a} 70 \mathrm{~kg}$ adult. The $0.32 \mu \mathrm{g} / \mathrm{ml}$ dose of PRZ corresponds to approximately $1.6 \mathrm{mg}$ PRZ for a $70 \mathrm{~kg}$ adult. The $2.4 \mu \mathrm{g} / \mathrm{ml}$ dose of AMD corresponds to approximately $12 \mathrm{mg}$ AMD for a $70 \mathrm{~kg}$ adult. The $4 \mu \mathrm{g} / \mathrm{ml}$ dose of SPD corresponds to approximately $20 \mathrm{mg}$ SPD for a $70 \mathrm{~kg}$ adult.

Lymphocyte primary cultures were set up by adding 11 drops of heparinized whole blood to $5 \mathrm{ml}$ of cell culture medium RPMI 1640 supplemented with $20 \%$ fetal calf serum, $0.63 \%$ L -glutamine, $0.63 \%$ penicillin/streptomycin and $2 \%$.

At the beginning of culture life, we added $5 \mu \mathrm{g} / \mathrm{ml}$ of 5-bromodeoxyuridine (BrdU) and the drug solutions. During the experiment, all cultures were maintained in the dark to minimize photolysis of $\mathrm{BrdU}$. The cultures were incubated for $72 \mathrm{~h}$ at $37^{\circ} \mathrm{C}$ and $0.3 \mu \mathrm{g} / \mathrm{ml}$ colchicine was added $2 \mathrm{~h}$ before collection by centrifugation. Air-dried preparations were stained by the fluorescence-plus Giemsa procedure and were scored for cells undergoing metaphase of the first mitotic cycle (both chromatids stained dark), second mitotic cycle (one chromatid of each chromosome stained dark) and third and/or subsequent mitotic cycles (a proportion of chromosomes with both chromatids stained light).

For SCEs, 30 suitably spread second-division metaphases from each culture were blindly scored. Each SCE value represents the mean + SEM. For PRI, at least 100 cells in the 1st, 2nd, 3rd and higher divisions from each culture were blindly scored. $\left.P R I=\left(M_{1}+2 M_{2}+3 M_{3}+\right) / 100\right)$, where $M_{1}, M_{2}$, and $M_{3}{ }^{+}$are the percent values of cells in the 1 st, 2 nd, and 3 rd and higher divisions, respectively. For the cultures' MI evaluation, we estimated the percentage of the total number of cells undergoing cell divisions to the total number of cells visible in the microslides. Student's $\mathrm{t}$-test $(\mathrm{p}<0.05)$ was performed to determine whether any of the SCE values calculated differed significantly from the controls and the $\mathrm{x}^{2}$-test was used for the MI and cell kinetic comparisons (PRI).

\section{Results}

As a positive control we used mitomycin $\mathrm{C}$ at $3 \mathrm{ng} / \mathrm{ml}$ with more than a doubling cytogenetic effect.

\section{Levomepromazine}

Levomepromazine showed a dose-dependent increase in the SCE frequency of lymphocytes, which became statistically significant (after $72 \mathrm{~h}$ of incubation at $37^{\circ} \mathrm{C}$ ) at $2.5 \mu \mathrm{g} / \mathrm{ml}$ (Table A.1). The PRI (Table A.2) and MI (Table A.3) values of the lymphocyte cultures were diminished by LVZ's effect, and the decrease became statistically significant at $2.5 \mu \mathrm{g} / \mathrm{ml}$.

Correlation between SCEs and PRI induction: Our findings indicate strong negative linear correlation between the SCEs and PRI increase induced by LVZ. Higher doses of LVZ are highly correlated
Table A.1: Induction of SCEs by LVZ in normal human lymphocyte cultures

\begin{tabular}{|c|c|c|c|c|}
\hline \multicolumn{5}{|c|}{ SCEs \pm SE/cell } \\
\hline $\begin{array}{c}\text { LVZ dose }(\mu \mathrm{g} / \\
\mathrm{ml})\end{array}$ & Donor A & Donor B & Donor C & Mean \\
\hline $0(\mathrm{control})$ & $7.60 \pm 0.820$ & $8.25 \pm 0.966$ & $6.75 \pm 1.551$ & $7.533 \pm 0.752$ \\
\hline $0.5 \mu \mathrm{g} / \mathrm{ml}$ & $8.05 \pm 1.276$ & $8.40 \pm 0.882$ & $7.35 \pm 1.039$ & $7.933 \pm 0.535$ \\
\hline $2.5 \mu \mathrm{g} / \mathrm{ml}$ & $8.35 \pm 0.988^{\mathrm{b}}$ & $8.95 \pm 1.316^{\mathrm{a}}$ & $7.80 \pm 1.472^{\mathrm{b}}$ & $8.367 \pm 0.575^{\mathrm{b}}$ \\
\hline $5 \mu \mathrm{g} / \mathrm{ml}$ & $8.45 \pm 0.944^{\mathrm{c}}$ & $9.35 \pm 1.424^{\mathrm{b}}$ & $8.60 \pm 1.391^{\mathrm{d}}$ & $8.800 \pm 0.482^{\mathrm{c}}$ \\
\hline $10 \mu \mathrm{g} / \mathrm{ml}$ & $8.85 \pm 1.039^{\mathrm{d}}$ & $9.55 \pm 1.605^{\mathrm{c}}$ & $9.05 \pm 1.637^{\mathrm{e}}$ & $9.150 \pm 0.361^{\mathrm{d}}$ \\
\hline $20 \mu \mathrm{g} / \mathrm{ml}$ & $9.45 \pm 1.700^{\mathrm{e}}$ & $9.95 \pm 1.669^{\mathrm{d}}$ & $10.40 \pm 1.635^{\mathrm{g}}$ & $9.933 \pm 0.475^{\mathrm{e}}$ \\
\hline $30 \mu \mathrm{g} / \mathrm{ml}$ & $10.0 \pm 2.127^{\mathrm{e}}$ & $10.45 \pm 2.139^{\mathrm{d}}$ & $10.70 \pm 1.525^{\mathrm{g}}$ & $10.38 \pm 0.355^{\mathrm{e}}$ \\
\hline
\end{tabular}

${ }^{a}$ Statistically significant difference $(p<0.1)$ compared to control

${ }^{b}$ Statistically significant difference $(p<0.05)$ compared to control

c Statistically significant difference $(p<0.01)$ compared to control

${ }^{d}$ Statistically significant difference $(p<0.005)$ compared to control

e Statistically significant difference $(p<0.001)$ compared to control

${ }^{f}$ Statistically significant difference $(p<0.0005)$ compared to control

${ }^{g}$ Statistically significant difference $(p<0.0001)$ compared to control

Table A.2: Decrease of PRI by LVZ in normal human lymphocyte cultures

\begin{tabular}{|c|c|c|c|c|}
\hline \multicolumn{5}{|c|}{ PRI } \\
\hline LVZ dose ( $\mu \mathrm{g} / \mathbf{m l})$ & Donor A & Donor B & Donor C & Mean \\
\hline 0 (control) & 1.83 & 1.74 & 1.85 & $1.807 \pm 0.059$ \\
\hline $0.5 \mu \mathrm{g} / \mathrm{ml}$ & $1.68^{\mathrm{a}}$ & $1.61^{\mathrm{a}}$ & 1.76 & $1.683 \pm 0.075^{\mathrm{a}}$ \\
\hline $2.5 \mu \mathrm{g} / \mathrm{ml}$ & $1.66^{\mathrm{b}}$ & $1.56^{\mathrm{b}}$ & $1.72^{\mathrm{a}}$ & $1.647 \pm 0.081^{\mathrm{b}}$ \\
\hline $5 \mu \mathrm{g} / \mathrm{ml}$ & $1.61^{\mathrm{c}}$ & $1.54^{\mathrm{c}}$ & $1.70^{\mathrm{b}}$ & $1.617 \pm 0.080^{\mathrm{c}}$ \\
\hline $10 \mu \mathrm{g} / \mathrm{ml}$ & $1.59^{\mathrm{d}}$ & $1.53^{\mathrm{d}}$ & $1.65^{\mathrm{c}}$ & $1.590 \pm 0.060^{\mathrm{d}}$ \\
\hline $20 \mu \mathrm{g} / \mathrm{ml}$ & $1.57^{\mathrm{d}}$ & $1.50^{\mathrm{d}}$ & $1.62^{\mathrm{d}}$ & $1.563 \pm 0.060^{\mathrm{d}}$ \\
\hline $30 \mu \mathrm{g} / \mathrm{ml}$ & $1.54^{\mathrm{e}}$ & $1.45^{\mathrm{e}}$ & $1.60^{\mathrm{d}}$ & $1.530 \pm 0.075^{\mathrm{e}}$ \\
\hline
\end{tabular}

a Statistically significant difference $(p<0.1)$ compared to control

${ }^{b}$ Statistically significant difference $(p<0.05)$ compared to control

Statistically significant difference $(p<0.01)$ compared to control

${ }^{d}$ Statistically significant difference $(p<0.005)$ compared to control

${ }^{e}$ Statistically significant difference $(p<0.001)$ compared to control

Table A.3: Decrease of MI by LVZ in normal human lymphocyte cultures

\begin{tabular}{|c|c|c|c|c|}
\hline \multicolumn{5}{|c|}{ MI } \\
\hline LVZ dose $(\mu \mathrm{g} / \mathrm{ml})$ & Donor A & Donor B & Donor C & Mean \\
\hline $0($ control) & $6.11 \%$ & $5.38 \%$ & $4.96 \%$ & $5.466 \% \pm 0.574$ \\
\hline $0.5 \mu \mathrm{g} / \mathrm{ml}$ & $4.37 \%$ & $4.62 \%$ & $4.27 \%$ & $4.374 \% \pm 0.965^{\mathrm{a}}$ \\
\hline $2.5 \mu \mathrm{g} / \mathrm{ml}$ & $4.01 \%$ & $4.17 \%$ & $3.92 \%$ & $4.091 \% \pm 0.488^{\mathrm{b}}$ \\
\hline $5 \mu \mathrm{g} / \mathrm{ml}$ & $3.62 \%$ & $4.02 \%$ & $3.64 \%$ & $3.782 \% \pm 0.552^{\mathrm{b}}$ \\
\hline $10 \mu \mathrm{g} / \mathrm{ml}$ & $3.09 \%$ & $3.91 \%$ & $3.31 \%$ & $3.417 \% \pm 0.473^{\mathrm{c}}$ \\
\hline $20 \mu \mathrm{g} / \mathrm{ml}$ & $2.73 \%$ & $3.61 \%$ & $3.15 \%$ & $3.142 \% \pm 0.736^{\mathrm{d}}$ \\
\hline $30 \mu \mathrm{g} / \mathrm{ml}$ & $2.18 \%$ & $3.12 \%$ & $2.96 \%$ & $2.883 \% \pm 0.836^{\mathrm{c}}$ \\
\hline
\end{tabular}

a Statistically significant difference $(p<0.1)$ compared to control

${ }^{b}$ Statistically significant difference $(p<0.05)$ compared to control

${ }^{c}$ Statistically significant difference $(p<0.01)$ compared to control

${ }^{d}$ Statistically significant difference $(p<0.005)$ compared to control

e Statistically significant difference $(p<0.001)$ compared to control

with higher SCE frequencies and lower PRI values. Pearson correlation coefficient $\mathrm{r}$ was estimated at $\mathrm{r}=0.9261$ with strong statistical significance $(\mathrm{p}<0.01)$.

\section{Perphenazine}

Perphenazine showed a dose-dependent increase in the SCE frequency of lymphocytes, which became statistically significant (after $72 \mathrm{~h}$ of incubation at $37^{\circ} \mathrm{C}$ ) at $0.64 \mu \mathrm{g} / \mathrm{ml}$ (Table B.1). The PRI (Table B.2) and MI (Table B.3) values of the lymphocyte cultures were diminished by PRZ's effect, and the decrease became statistically significant at 2.56 and $1.28 \mu \mathrm{g} / \mathrm{ml}$ respectively.

Correlation between SCEs and PRI induction: Our findings indicate strong negative linear correlation between the SCEs and PRI 
Table B.1: Induction of SCEs by PRZ in normal human lymphocyte cultures

\begin{tabular}{|c|c|c|c|c|}
\hline \multicolumn{5}{|c|}{ SCEs \pm SE/cell } \\
\hline PRZ dose ( $\mu \mathrm{g} / \mathrm{ml})$ & Donor A & Donor B & Donor C & Mean \\
\hline $0($ control) & $7.60 \pm 0.8207$ & $7.10 \pm 1.4473$ & $6.85 \pm 1.4608$ & $7.18 \pm 0.382$ \\
\hline $0.32 \mu \mathrm{g} / \mathrm{ml}$ & $7.90 \pm 0.7591$ & $7.50 \pm 1.1470$ & $7.20 \pm 1.2396$ & $7.53 \pm 0.351^{\mathrm{a}}$ \\
\hline $0.64 \mu \mathrm{g} / \mathrm{ml}$ & $8.35 \pm 1.2258^{\mathrm{b}}$ & $7.70 \pm 1.2607$ & $7.70 \pm 1.0310^{\mathrm{b}}$ & $7.91 \pm 0.375^{\mathrm{b}}$ \\
\hline $1.28 \mu \mathrm{g} / \mathrm{ml}$ & $8.85 \pm 1.2258^{\mathrm{d}}$ & $8.55 \pm 1.3945^{\mathrm{c}}$ & $8.35 \pm 1.2258^{\mathrm{d}}$ & $8.58 \pm 0.252^{\mathrm{d}}$ \\
\hline $2.56 \mu \mathrm{g} / \mathrm{ml}$ & $9.30 \pm 1.4179^{\mathrm{e}}$ & $9.15 \pm 1.5652^{\mathrm{e}}$ & $9.10 \pm 1.4832^{\mathrm{f}}$ & $9.18 \pm 0.104^{\mathrm{e}}$ \\
\hline $3.84 \mu \mathrm{g} / \mathrm{ml}$ & $10.20 \pm 1.0563^{\mathrm{g}}$ & $9.70 \pm 1.4179^{\mathrm{f}}$ & $10.35 \pm 1.6311^{\mathrm{g}}$ & $10.08 \pm 0.340^{\mathrm{g}}$ \\
\hline $5.12 \mu \mathrm{g} / \mathrm{ml}$ & $10.65 \pm 1.5035^{\mathrm{g}}$ & $10.65 \pm 1.4964^{\mathrm{g}}$ & $10.70 \pm 1.7501^{\mathrm{g}}$ & $10.67 \pm 0.029^{\mathrm{g}}$ \\
\hline
\end{tabular}

${ }^{a}$ Statistically significant difference $(p<0.1)$ compared to control

${ }^{b}$ Statistically significant difference $(p<0.05)$ compared to control

${ }^{c}$ Statistically significant difference $(p<0.01)$ compared to control

${ }^{d}$ Statistically significant difference $(p<0.005)$ compared to control

${ }^{e}$ Statistically significant difference $(p<0.001)$ compared to control

${ }^{f}$ Statistically significant difference $(p<0.0005)$ compared to control

${ }^{g}$ Statistically significant difference $(p<0.0001)$ compared to control

Table B.2: Decrease of PRI by PRZ in normal human lymphocyte cultures

\begin{tabular}{|c|c|c|c|c|}
\hline \multicolumn{5}{|c|}{ PRI } \\
\hline PRZ dose $(\mu \mathrm{g} / \mathrm{ml})$ & Donor A & Donor B & Donor C & Mean \\
\hline $0($ control) & 1.7 & 1.78 & 1.75 & $1.743 \pm 0.138$ \\
\hline $0.32 \mu \mathrm{g} / \mathrm{ml}$ & 1.69 & 1.72 & 1.73 & $1.717 \pm 0.082$ \\
\hline $0.64 \mu \mathrm{g} / \mathrm{ml}$ & 1.64 & 1.67 & 1.68 & $1.674 \pm 0.103$ \\
\hline $1.28 \mu \mathrm{g} / \mathrm{ml}$ & 1.59 & $1.64^{\mathrm{b}}$ & $1.62^{\mathrm{a}}$ & $1.628 \pm 0.129^{\mathrm{a}}$ \\
\hline $2.56 \mu \mathrm{g} / \mathrm{ml}$ & $1.53^{\mathrm{b}}$ & $1.60^{\mathrm{b}}$ & $1.59^{\mathrm{b}}$ & $1.584 \pm 0.093^{\mathrm{b}}$ \\
\hline $3.84 \mu \mathrm{g} / \mathrm{ml}$ & $1.52^{\mathrm{c}}$ & $1.58^{\mathrm{c}}$ & $1.55^{\mathrm{d}}$ & $1.552 \pm 0.110^{\mathrm{c}}$ \\
\hline $5.12 \mu \mathrm{g} / \mathrm{ml}$ & $1.49^{\mathrm{d}}$ & $1.57^{\mathrm{d}}$ & $1.52^{\mathrm{d}}$ & $1.537 \pm 0.133^{\mathrm{d}}$ \\
\hline
\end{tabular}

a Statistically significant difference $(p<0.1)$ compared to control

${ }^{b}$ Statistically significant difference $(p<0.05)$ compared to control

Statistically significant difference $(p<0.01)$ compared to control

${ }^{d}$ Statistically significant difference $(p<0.005)$ compared to control

Table B.3: Decrease of MI by PRZ in normal human lymphocyte cultures

\begin{tabular}{|c|c|c|c|c|}
\hline \multicolumn{5}{|c|}{ MI } \\
\hline PRZ dose $(\boldsymbol{\mu g} / \mathbf{m l})$ & Donor A & Donor B & Donor C & Mean \\
\hline 0 (control) & $5.27 \%$ & $5.61 \%$ & $5.38 \%$ & $5.428 \% \pm 0.463$ \\
\hline $0.32 \mu \mathrm{g} / \mathrm{ml}$ & $5.03 \%$ & $5.03 \%$ & $5.11 \%$ & $5.085 \% \pm 0.386$ \\
\hline $0.64 \mu \mathrm{g} / \mathrm{ml}$ & $4.51 \%$ & $4.62 \%$ & $4.73 \%$ & $4.624 \% \pm 0.447^{\mathrm{a}}$ \\
\hline $1.28 \mu \mathrm{g} / \mathrm{ml}$ & $3.92 \%$ & $4.30 \%$ & $4.29 \%$ & $4.189 \% \pm 0.352^{\mathrm{b}}$ \\
\hline $2.56 \mu \mathrm{g} / \mathrm{ml}$ & $3.47 \%$ & $3.96 \%$ & $3.97 \%$ & $3.817 \% \pm 0.549^{\mathrm{b}}$ \\
\hline $3.84 \mu \mathrm{g} / \mathrm{ml}$ & $3.28 \%$ & $3.72 \%$ & $3.61 \%$ & $3.525 \% \pm 0.436^{\mathrm{c}}$ \\
\hline $5.12 \mu \mathrm{g} / \mathrm{ml}$ & $3.06 \%$ & $3.30 \%$ & $3.22 \%$ & $3.217 \% \pm 0.419^{\mathrm{d}}$ \\
\hline
\end{tabular}

${ }^{a}$ Statistically significant difference $(p<0.1)$ compared to control

${ }^{b}$ Statistically significant difference $(p<0.05)$ compared to control

${ }^{c}$ Statistically significant difference $(p<0.01)$ compared to control

${ }^{d}$ Statistically significant difference $(p<0.005)$ compared to control

increase induced by PRZ. Higher doses of PRZ are highly correlated with higher SCE frequencies and lower PRI values. Pearson correlation coefficient $r$ was estimated at -0.9795 with strong statistical significance $(\mathrm{p}<0.001)$.

\section{Amisulpride}

Amisulpride showed a dose-dependent increase in the SCE frequency of lymphocytes, which became statistically significant (after $72 \mathrm{~h}$ of incubation at $37^{\circ} \mathrm{C}$ ) at $9.6 \mu \mathrm{g} / \mathrm{ml}$ (Table C.1). The PRI (Table C.2) and MI (Table C.3) values of the lymphocyte cultures were diminished by PRZ's effect, and the decrease became statistically significant at 9.6 and $19.2 \mu \mathrm{g} / \mathrm{ml}$ respectively.

Correlation between SCEs and PRI induction: Our findings indicate strong negative linear correlation between the SCEs and PRI increase induced by AMD. Higher doses of AMD are highly correlated with higher SCE frequencies and lower PRI values. Pearson correlation coefficient $r$ was estimated at -0.9953 with strong statistical significance $(\mathrm{p}<0.001)$.

\section{Sulpiride}

Sulpiride showed a dose-dependent increase in the SCE frequency of lymphocytes, which became statistically significant (after $72 \mathrm{~h}$ of incubation at $37^{\circ} \mathrm{C}$ ) at $8 \mu \mathrm{g} / \mathrm{ml}$ (Table D.1).The PRI (Table D.2) and MI (Table D.3) values of the lymphocyte cultures were diminished by SPD's effect, and the decrease became statistically significant at 8 and $16 \mu \mathrm{g} / \mathrm{ml}$ respectively.

Table C.1: Induction of SCEs by AMD in normal human lymphocyte cultures

\begin{tabular}{|c|c|c|c|c|}
\hline \multicolumn{5}{|c|}{ 1.SCEs \pm SE/cell } \\
\hline $\begin{array}{c}\text { AMD dose }(\mu \mathrm{g} / \\
\mathbf{m l})\end{array}$ & Donor A & Donor B & Donor C & Mean \\
\hline $0($ control) & $7.35 \pm 1.268$ & $6.85 \pm 0.7451$ & $7.95 \pm 1.3168$ & $7.383 \pm 0.963$ \\
\hline $2.4 \mu \mathrm{g} / \mathrm{ml}$ & $8.05 \pm 1.099^{\mathrm{a}}$ & $7.45 \pm 0.6863^{\mathrm{b}}$ & $8.25 \pm 1.3327$ & $7.917 \pm 1.042^{\mathrm{a}}$ \\
\hline $9.6 \mu \mathrm{g} / \mathrm{ml}$ & $8.35 \pm 0.875^{\mathrm{b}}$ & $8.10 \pm 0.9679^{\mathrm{e}}$ & $8.70 \pm 1.2607^{\mathrm{a}}$ & $8.383 \pm 0.967^{\mathrm{b}}$ \\
\hline $19.2 \mu \mathrm{g} / \mathrm{ml}$ & $8.70 \pm 1.341^{\mathrm{c}}$ & $8.65 \pm 1.1821^{\mathrm{f}}$ & $9.30 \pm 1.5927^{\mathrm{b}}$ & $8.883 \pm 1.285^{\mathrm{c}}$ \\
\hline $38.4 \mu \mathrm{g} / \mathrm{ml}$ & $9.05 \pm 1.316^{\mathrm{d}}$ & $8.75 \pm 1.1641^{\mathrm{g}}$ & $9.55 \pm 1.2763^{\mathrm{d}}$ & $9.117 \pm 1.304^{\mathrm{d}}$ \\
\hline $76.8 \mu \mathrm{g} / \mathrm{ml}$ & $9.70 \pm 1.174^{\mathrm{g}}$ & $9.65 \pm 1.0399^{\mathrm{g}}$ & $10.20 \pm 1.3992^{\mathrm{f}}$ & $9.850 \pm 1.218^{\mathrm{f}}$ \\
\hline $115.2 \mu \mathrm{g} / \mathrm{ml}$ & $10.15 \pm 1.565^{\mathrm{g}}$ & $9.95 \pm 1.1909^{\mathrm{g}}$ & $10.90 \pm 1.8035^{\mathrm{g}}$ & $10.33 \pm 1.536^{\mathrm{g}}$ \\
\hline
\end{tabular}

${ }^{a}$ Statistically significant difference $(p<0.1)$ compared to control

${ }^{b}$ Statistically significant difference $(p<0.05)$ compared to control

${ }^{c}$ Statistically significant difference $(p<0.01)$ compared to control

${ }^{d}$ Statistically significant difference $(p<0.005)$ compared to control

${ }^{e}$ Statistically significant difference $(p<0.001)$ compared to control

${ }^{f}$ Statistically significant difference $(p<0.0005)$ compared to control

${ }^{g}$ Statistically significant difference $(p<0.0001)$ compared to control

Table C.2: Decrease of PRI by AMD in normal human lymphocyte cultures

\begin{tabular}{|c|c|c|c|c|}
\hline \multicolumn{5}{|c|}{ PRI } \\
\hline AMD dose $(\boldsymbol{\mu g} / \mathbf{m l})$ & Donor A & Donor B & Donor C & Mean \\
\hline 0 (control) & 2.09 & 2.04 & 1.79 & $1.973 \pm 0.161$ \\
\hline $2.4 \mu \mathrm{g} / \mathrm{ml}$ & 1.99 & $1,92^{\mathrm{a}}$ & 1.72 & $1.877 \pm 0.140$ \\
\hline $9.6 \mu \mathrm{g} / \mathrm{ml}$ & $1,93^{\mathrm{b}}$ & $1.85^{\mathrm{d}}$ & $1.63^{\mathrm{b}}$ & $1.803 \pm 0.155^{\mathrm{b}}$ \\
\hline $19.2 \mu \mathrm{g} / \mathrm{ml}$ & $1.89^{\mathrm{d}}$ & $1.75^{\mathrm{e}}$ & $1.60^{\mathrm{d}}$ & $1.747 \pm 0.145^{\mathrm{d}}$ \\
\hline $38.4 \mu \mathrm{g} / \mathrm{ml}$ & $1.82^{\mathrm{e}}$ & $1.61^{\mathrm{e}}$ & $1.57^{\mathrm{d}}$ & $1.667 \pm 0.134^{\mathrm{e}}$ \\
\hline $76.8 \mu \mathrm{g} / \mathrm{ml}$ & $1.71^{\mathrm{e}}$ & $1.50^{\mathrm{e}}$ & $1.48^{\mathrm{e}}$ & $1.563 \pm 0.127^{\mathrm{e}}$ \\
\hline $115.2 \mu \mathrm{g} / \mathrm{ml}$ & $1.60^{\mathrm{e}}$ & $1.38^{\mathrm{e}}$ & $1.34^{\mathrm{e}}$ & $1.440 \pm 0.140^{\mathrm{e}}$ \\
\hline
\end{tabular}

a Statistically significant difference $(p<0.1)$ compared to control

${ }^{b}$ Statistically significant difference $(p<0.05)$ compared to control

Statistically significant difference $(p<0.01)$ compared to control

${ }^{d}$ Statistically significant difference $(p<0.005)$ compared to control

e Statistically significant difference $(p<0.001)$ compared to control

Table C.3: Decrease of MI by AMD in normal human lymphocyte cultures

\begin{tabular}{|c|c|c|c|c|}
\hline \multicolumn{5}{|c|}{ MI } \\
\hline AMD dose $(\boldsymbol{\mu g} / \mathbf{m l})$ & Donor A & Donor B & Donor C & Mean \\
\hline 0 (control) & $4.80 \%$ & $6.35 \%$ & $5.63 \%$ & $5.593 \% \pm 0.776$ \\
\hline $2.4 \mu \mathrm{g} / \mathrm{ml}$ & $4.20 \%$ & $5.94 \%$ & $5.11 \%$ & $5.083 \% \pm 0.870$ \\
\hline $9.6 \mu \mathrm{g} / \mathrm{ml}$ & $3.99 \%$ & $5.22 \%$ & $4.69 \%$ & $4.633 \% \pm 0.617^{\mathrm{a}}$ \\
\hline $19.2 \mu \mathrm{g} / \mathrm{ml}$ & $3.08 \%$ & $4.37 \%$ & $4.42 \%$ & $3.956 \% \pm 0.759^{\mathrm{b}}$ \\
\hline $38.4 \mu \mathrm{g} / \mathrm{ml}$ & $2.73 \%$ & $3.10 \%$ & $4.07 \%$ & $3.300 \% \pm 0.692^{\mathrm{c}}$ \\
\hline $76.8 \mu \mathrm{g} / \mathrm{ml}$ & $2.39 \%$ & $2.73 \%$ & $3.85 \%$ & $2.990 \% \pm 0.764^{\mathrm{d}}$ \\
\hline $115.2 \mu \mathrm{g} / \mathrm{ml}$ & $1.76 \%$ & $2.19 \%$ & $3.06 \%$ & $2.336 \% \pm 0.662^{\mathrm{e}}$ \\
\hline
\end{tabular}

a Statistically significant difference $(p<0.1)$ compared to control

${ }^{b}$ Statistically significant difference $(p<0.05)$ compared to control

${ }^{c}$ Statistically significant difference $(p<0.01)$ compared to control

${ }^{d}$ Statistically significant difference $(p<0.005)$ compared to control

e Statistically significant difference $(p<0.001)$ compared to control 
Table D.1: Induction of SCEs by SPD in normal human lymphocyte cultures

\begin{tabular}{|c|c|c|c|c|}
\hline \multicolumn{5}{|c|}{ SCEs \pm SE/cell } \\
\hline SPD dose $(\boldsymbol{\mu g} / \mathbf{m l})$ & Donor A & Donor B & Donor C & Mean \\
\hline 0 (control) & $7.45 \pm 1.0990$ & $7.90 \pm 1.5525$ & $6.85 \pm 1.4608$ & $7.400 \pm 0.527$ \\
\hline $4 \mu \mathrm{g} / \mathrm{ml}$ & $7.75 \pm 1.1180$ & $7.95 \pm 0.8255$ & $7.60 \pm 1.3138$ & $7.767 \pm 0.176^{\mathrm{a}}$ \\
\hline $8 \mu \mathrm{g} / \mathrm{ml}$ & $8.25 \pm 1.2926^{\mathrm{b}}$ & $9.05 \pm 1.0500^{\mathrm{b}}$ & $8.20 \pm 1.1964^{\mathrm{b}}$ & $8.500 \pm 0.477^{\mathrm{b}}$ \\
\hline $16 \mu \mathrm{g} / \mathrm{ml}$ & $8.85 \pm 1.4244^{\mathrm{d}}$ & $9.60 \pm 1.0462^{\mathrm{d}}$ & $8.65 \pm 0.9333^{\mathrm{e}}$ & $9.033 \pm 0.501^{\mathrm{d}}$ \\
\hline $32 \mu \mathrm{g} / \mathrm{ml}$ & $10.15 \pm 1.7851^{\mathrm{f}}$ & $10.30 \pm 1.6575^{\mathrm{f}}$ & $9.90 \pm 1.9973^{\mathrm{f}}$ & $10.12 \pm 0.202^{\mathrm{f}}$ \\
\hline $64 \mu \mathrm{g} / \mathrm{ml}$ & $11.35 \pm 1.6630^{\mathrm{g}}$ & $10.75 \pm 1.6181^{\mathrm{f}}$ & $11.05 \pm 1.8488^{\mathrm{g}}$ & $11.05 \pm 0.300^{\mathrm{g}}$ \\
\hline $96 \mu \mathrm{g} / \mathrm{ml}$ & $12.10 \pm 1.8324^{\mathrm{g}}$ & $11.20 \pm 1.5078^{\mathrm{g}}$ & $11.95 \pm 2.0641^{\mathrm{g}}$ & $11.75 \pm 0.482^{\mathrm{g}}$ \\
\hline
\end{tabular}

${ }^{a}$ Statistically significant difference $(p<0.1)$ compared to control

${ }^{b}$ Statistically significant difference $(p<0.05)$ compared to control

${ }^{c}$ Statistically significant difference $(p<0.01)$ compared to control

${ }^{d}$ Statistically significant difference $(p<0.005)$ compared to control

${ }^{e}$ Statistically significant difference $(p<0.001)$ compared to control

${ }^{f}$ Statistically significant difference $(p<0.0005)$ compared to control

${ }^{g}$ Statistically significant difference $(p<0.0001)$ compared to control

Table D.2: Decrease of PRI by SPD in normal human lymphocyte cultures

\begin{tabular}{|c|c|c|c|c|}
\hline \multicolumn{5}{|c|}{ PRI } \\
\hline SPD dose ( $\boldsymbol{\mu g} / \mathbf{m l})$ & Donor A & Donor B & Donor C & Mean \\
\hline 0 (control) & 1.70 & 1.83 & 1.75 & $1.760 \pm 0.066$ \\
\hline $4 \mu \mathrm{g} / \mathrm{ml}$ & 1.69 & 1.76 & 1.71 & $1.724 \pm 0.091^{\mathrm{a}}$ \\
\hline $8 \mu \mathrm{g} / \mathrm{ml}$ & 1.62 & $1.69^{\mathrm{b}}$ & $1.60^{\mathrm{b}}$ & $1.638 \pm 0.108^{\mathrm{b}}$ \\
\hline $16 \mu \mathrm{g} / \mathrm{ml}$ & $1.56^{\mathrm{b}}$ & $1.65^{\mathrm{c}}$ & $1.56^{\mathrm{c}}$ & $1.582 \pm 0.137^{\mathrm{c}}$ \\
\hline $32 \mu \mathrm{g} / \mathrm{ml}$ & $1.50^{\mathrm{c}}$ & $1.62^{\mathrm{d}}$ & $1.49^{\mathrm{e}}$ & $1.535 \pm 0.081^{\mathrm{d}}$ \\
\hline $64 \mu \mathrm{g} / \mathrm{ml}$ & $1.46^{\mathrm{e}}$ & $1.56^{\mathrm{e}}$ & $1.43^{\mathrm{e}}$ & $1.486 \pm 0.137^{\mathrm{e}}$ \\
\hline $96 \mu \mathrm{g} / \mathrm{ml}$ & $1.42^{\mathrm{e}}$ & $1.49^{\mathrm{e}}$ & $1.39^{\mathrm{e}}$ & $1.441 \pm 0.119^{\mathrm{c}}$ \\
\hline
\end{tabular}

a Statistically significant difference $(p<0.1)$ compared to control

${ }^{b}$ Statistically significant difference $(p<0.05)$ compared to control

${ }^{c}$ Statistically significant difference $(p<0.01)$ compared to control

${ }^{d}$ Statistically significant difference $(p<0.005)$ compared to control

e Statistically significant difference $(p<0.001)$ compared to control

Table D.3: Decrease of MI by SPD in normal human lymphocyte cultures

\begin{tabular}{|c|c|c|c|c|}
\hline \multicolumn{5}{|c|}{ MI } \\
\hline SPD dose $(\boldsymbol{\mu g} / \mathbf{m l})$ & Donor A & Donor B & Donor C & Mean \\
\hline 0 (control) & $4.74 \%$ & $5.54 \%$ & $5.12 \%$ & $5.127 \% \pm 0.463$ \\
\hline $4 \mu \mathrm{g} / \mathrm{ml}$ & $4.51 \%$ & $5.12 \%$ & $4.85 \%$ & $4.817 \% \pm 0.537$ \\
\hline $8 \mu \mathrm{g} / \mathrm{ml}$ & $3.96 \%$ & $4.68 \%$ & $4.28 \%$ & $4.381 \% \pm 0.479^{\mathrm{a}}$ \\
\hline $16 \mu \mathrm{g} / \mathrm{ml}$ & $3.49 \%$ & $4.28 \%$ & $3.97 \%$ & $3.922 \% \pm 0.537^{\mathrm{b}}$ \\
\hline $32 \mu \mathrm{g} / \mathrm{ml}$ & $3.05 \%$ & $4.01 \%$ & $3.51 \%$ & $3.574 \% \pm 0.475^{\mathrm{c}}$ \\
\hline $64 \mu \mathrm{g} / \mathrm{ml}$ & $2.70 \%$ & $3.58 \%$ & $2.82 \%$ & $3.059 \% \pm 0.649^{\mathrm{d}}$ \\
\hline $96 \mu \mathrm{g} / \mathrm{ml}$ & $2.21 \%$ & $2.93 \%$ & $2.33 \%$ & $2.487 \% \pm 0.588^{\mathrm{d}}$ \\
\hline
\end{tabular}

${ }^{a}$ Statistically significant difference $(p<0.1)$ compared to control

${ }^{b}$ Statistically significant difference $(p<0.05)$ compared to control

${ }^{c}$ Statistically significant difference $(p<0.01)$ compared to control

${ }^{d}$ Statistically significant difference $(p<0.005)$ compared to control

Correlation between SCEs and PRI induction: Our findings indicate strong negative linear correlation between the SCEs and PRI increase induced by SPD. Higher doses of SPD are highly correlated with higher SCE frequencies and lower PRI values. Pearson correlation coefficient $r$ was estimated at -0.982 with strong statistical significance $(\mathrm{p}<0.001)$.

\section{Discussion}

Since typical and atypical antipsychotics are regarded as potential antineoplastics, the ability of LVP, PRZ, AMD and SPD to induce genotoxic and cytostatic effects was tested in cultured normal human lymphocytes $[18,19]$. Human lymphocytes constitute a possible dosimeter of exposure to genotoxic antineoplastic agents [34]. All the chemicals tested demonstrate a strong correlation between SCE induction and PRI suppression as well as an ability to suppress significantly the MI.
SCE induced by certain agents upon normal cells in vitro are positively correlated with in vivo tumor response to these agents. Unrepaired DNA damage expressed as SCE induced by phenothiazines (LVZ, PRZ) or substituted benzamide derivatives (AMD, SPD) in normal cells may indicate inability to repair damage in cancer cells since both cell types have similar DNA repair mechanisms [34]. Other studies investigating a relationship between SCE induction and other expressions of genotoxicity have shown a positive relationship between SCE induction, alteration in cell cycle kinetics, reduced cell survival and antitumor activity [34]. In the present study a strong correlation between SCE induction and PRI depression in the four antipsychotics tested was observed. It is worth mentioning that SPD along with clozapine has been reported as a demethylating agent [32] Hypomethylation of DNA is known as producing difficulties for the cellular enzymes in discriminating between the parental and the newly formed DNA strand [36]. The presence of unrepaired lesions at this level may give rise in error-prone ligation, resulting in SCE induction cell division delays and MI suppression.

AMS and SPD are two substituted benzamide derivatives. Benzamide interferes with DNA repair by inhibiting poly(ADPribose)polymerase (PADPRP) and preventing the depletion of NAD to polyADP-ribose. PolyADP-ribose participates in DNA repair [37]. SCE have been reported as highly sensitive indicators of DNA damage and/or subsequent repair and as an important biomarker for predicting cancer chemoresponse [34]. It must be also mentioned that phenothiazines have been reported to: a) induce apoptosis in vitro [38] b) inhibit cell division of glioma cells in human neuroblastoma [39] and c) induce apoptosis in T-cell acute lymphoblastic leukemia [40].

\section{Conclusion}

In conclusion, the genotoxic (elevation of SCE) and cytostatic/ cytotoxic (suppression of PRI and MI) effects along with the previously reported induction of apoptosis and inhibition of DNA repair by the antipsychotics tested suggest their consideration as agents with possible clinical antineoplastic application.

\section{Acknowledgements:}

\section{Declarations of interest: None.}

Funding: This research did not receive any specific grant from funding agencies in the public, commercial, or not-for-profit sectors.

\section{References}

1. Hellenic National Formulary (2007) Drugs for the Central Nervous SystemAntipsychotics. NOD: 197-200.

2. Hellenic National Formulary (2007) Drugs for the Central Nervous System, Levomepromazine. NOD: 204.

3. National Center for Biotechnology Information (2018) PubChem Compound Database; $\mathrm{CID}=72287$.

4. Lal S, Nair NP, Cecyre D, Quirion R (1993) Levomepromazine receptor binding profile in human brain-implications for treatment-resistant schizophrenia. Acta Psychiatr Scand 87: 380-383. [Crossref]

5. Sivaraman P, Rattehalli RD, Jayaram MB (2010) Levomepromazine for schizophrenia Cochrane Database Syst Rev 6: CD007779. [Crossref]

6. Galinos-Guide for drugs. [https://www.galinos.gr/web/drugs/main/substances/ perphenazine]. Accessed January 2020

7. National Center for Biotechnology Information (2018) PubChem Compound Database; $\mathrm{CID}=4748$

8. Sweet RA, Pollock BG, Mulsant BH, Rosen j, Sorisio D, et al. (2000) Pharmacologic Profile of Perphenazine's Metabolites. J Clin Psychopharmacol 20: 181-187. [Crossref] 
9. Hellenic National Formulary (2007) Drugs for the Central Nervous System, Amisulpride. NOD: 201.

10. Perrault G, Depoortere R, Morel E, Sanger DJ, Scatton B (1997) Psychopharmacological profile of amisulpride: an antipsychotic drug with presynaptic D2/D3 dopamine receptor antagonist activity and limbic selectivity. J Pharmacol Exp Ther 280: 73-82. [Crossref]

11. National Center for Biotechnology Information (2018) PubChem Compound Database; $\mathrm{CID}=2159$

12. Green B (2002) Focus on Amisulpride. Curr Med Res Opin 18: 113-117. [Crossref]

13. Tu MC, Hsiao CC (2011) Amisulpride and neuroleptic malignant syndrome. Chang Gung Med J 34: 536-540. [Crossref]

14. Hellenic National Formulary (2007) Drugs for the Central Nervous System, Sulpiride. NOD: 209

15. Lai ECC, Chang CH, Yang YHK, Lin SJ, Lin CY (2013) Effectiveness of Sulpiride in Adult Patients With Schizophrenia. Schizoph Bull 39: 673-683. [Crossref]

16. Maitre M, Ratomponirina C, Gobaille S, Hodé Y, Hechler V (1994) Displacement of $[3 \mathrm{H}]$ gamma-hydroxybutyrate binding by benzamide neuroleptics and prochlorperazine but not by other antipsychotics. Eur J Pharmacol 256: 211-214. [Crossref]

17. National Center for Biotechnology Information (2018) PubChem Compound Database; $\mathrm{CID}=5355$.

18. Nordenberg J, Fenig E, Landau M, Weizman R, Weizman A (1999) Effects of psychotropic drugs on cell proliferation and differentiation. Biochem Pharmacol 58: 1229-1236. [Crossref]

19. Brambilla G, Mattioli F, Martelli A (2009) Genotoxic and carcinogenic effects of antipsychotics and antidepressants. Toxicology 261: 77-88. [Crossref]

20. Karapidaki I, Ekonomopoulou MT, Akritopoulou K, Anestakis D, Iakovidou-Kritsi Z (2011) Cytogenetic effects of valproic acid and ziprasidone in human lymphocyte cultures. Neuropsychobiology 64: 219-223. [Crossref]

21. Zhelev Z, Ohba H, Bakalova R, Hadjimitova V, Ishikawa M, et al. (2004) Phenothiazines suppress proliferation and induce apoptosis in cultured leukemic cells without any influence on the viability of normal lymphocytes. Phenothiazines and leukemia. Cancer Chemother Pharmacol 53: 267-275. [Crossref]

22. Hodgson R, Wildgust HJ, Bushe CJ (2010) Cancer and schizophrenia: is there a paradox? J Psychopharmacol 24: 51-60. [Crossref]

23. Howard LM, Barley EA, Davies E, Rigg A, Lempp H, et al.(2010) Cancer diagnosis in people with severe mental illness: practical and ethical issues. Lancet Oncol 11: 797-804. [Crossref]

24. Fond G, Macgregor A, Attal J,Larue A, Brittner M, et al.(2012) Antipsychotic drugs: pro-cancer or anti-cancer? A systematic review. Med Hypotheses 79: 38-42. [Crossref]

25. Qi Lu, Ding YQ (2013) Potential antitumor mechanisms of phenothiazine drugs. Sci China Life Sci 56: 1020-1027. [Crossref]
26. Spengler G, Csonka Á, Molnar J, Amaral L (2016) The Anticancer Activity of the Old Neuroleptic Phenothiazine-type Drug Thioridazine. Anticancer Res 36: 5701-5706. [Crossref]

27. Mortensen PB (1992) Neuroleptic medication and reduced risk of prostate cancer in schizophrenic patients. Acta Psychiatr Scand 85: 390-3. [Crossref]

28. Lialiaris TS, Papachristou F, Mourelatos C, Simopoulou M (2009) Antineoplastic and cytogenetic effects of chlorpromazine on human lymphocytes in vitro and on Ehrlich ascites tumor cells in vivo. Anticancer Drugs 20: 746-751. [Crossref]

29. Yde CW, Clausen MP, Bennetzen MV, Lykkesfeldt AE, Mouritsen OG, et al.(2009) The antipsychotic drug chlorpromazine enhances the cytotoxic effect of tamoxifen in tamoxifen-sensitive and tamoxifen-resistant human breast cancer cells. Anticancer Drugs 20: 723-735. [Crossref]

30. Hartung B, Sampson S, Leucht S (2015) Perphenazine for schizophrenia. Cochrane Database Syst Rev 3: CD003443. [Crossref]

31. Madej A, Belowski D, Kowalski J, Herman ZS (1998) Influence of neuroleptics on cytotoxic activity of macrophages in rats. Boll Chim Farm 137: 135-139. [Crossref]

32. Dong E, Nelson M, Grayson DR, Costa E, Guidotti A (2008) Clozapine and sulpiride but not haloperidol or olanzapine activate brain DNA demethylation. Proc Natl Acad Sci U S A 105: 13614-13619. [Crossref]

33. Goulet K, Grignon S (2008) Case report: clozapine given in the context of chemotherapy for lung cancer. Psychooncology 17: 512-516. [Crossref]

34. Mourelatos D (2016) Sister chromatid exchange assay as a predictor of tumor chemoresponse. Mutat Res Genet Toxicol Environ Mutagen 803: 1-12. [Crossref]

35. Mourelatos D (1996) Chromosome study as predictor of chemoresponse of tumors. The Cancer J 9:136-141.

36. Ioannidou E, Lialiaris T, Mourelatos D, Dozi-Vassiliades J (1989) Synergistic induction of cytogenetic damage by alkylating antineoplastics and 5-azacytidine in human lymphocytes. Environ Mol Mutagen 4: 6-12. [Crossref]

37. Mourelatos D, Kourakis A, Koliouskas DE, Hatzitheodoridou P, Dozi-Vassiliades J (1986) Enhancement of cytogenetic damage by inhibitors of poly(ADP-ribose) polymerase in human lymphocytes exposed to antineoplastics in vivo and in vitro. Teratog Carcinog Mutagen 6: 485-492. [Crossref]

38. Motohashi N, Kurihara T, Satoh K, Sakagami H, Mucsi I, et al. (1999) Antitumor activity of benzo[a]phenothiazines. Anticancer Res 19: 1837-1842. [Crossref]

39. P.Eliopoulos, D.Mourelatos, J.Dozi-Vassiliades (1995) Comparative study on Salmonella mutagenicity and on cytogenetic and antineoplastic effects induced by cyclophosphamide and 3-aminobenzamide in cells of three transplantable tumors in vivo. Mut Res 342: 141-146. [Crossref]

40. Gutierrez A, Pan L, Groen R WJ, Baleydier F, Kentsis A (2014) Phenothiazines induce PP2A-mediated apoptosis in T cell acute lymphoblastic leukemia. J Clin Invest 124: 644-655. [Crossref]

Copyright: (C2020 Katsioulis EG. This is an open-access article distributed under the terms of the Creative Commons Attribution License, which permits unrestricted use, distribution, and reproduction in any medium, provided the original author and source are credited. 